\title{
An Unusual Case of Urinary Incontinence
}

\author{
Eric R. Anderson, Darius J. Unwala \\ Departments of ${ }^{1}$ General Surgery and Urology, Marshfield Clinic, Marshfield, Wisconsin \\ E-mail: unwala.darius@marshfieldclinic.org \\ Received January 18, 2011; revised February 16, 2011; accepted February 24, 2011
}

\begin{abstract}
Urinary incontinence and hematuria are frequent complaints evaluated by urologists often with readily identifiable etiologies. Occasionally, an unlikely source is found. We present an unusual case of urinary incontinence and hematuria in an elderly female. Evaluation revealed that the complaints were due to a large cyst emanating from her right hip which was the result of a previous right hip arthroplasty in 1970 with three subsequent revisions. Her symptoms resolved spontaneously and the cyst has remained unchanged.
\end{abstract}

Keywords: Hematuria, Hip Arthroplasty, Particle Disease, Synovial Cysts, Urinary Incontinence

\section{Introduction}

Urinary incontinence and hematuria are frequent complaints evaluated by urologists. In the majority of cases, the etiologies are readily identifiable. Occasionally these complaints arise from an unlikely source. We present an unusual case of sudden onset of urinary incontinence and hematuria in an elderly female.

\section{Case Report}

An 81-year-old female presented to her primary care provider in January 2009 with an acute onset of urinary incontinence, hematuria, and suprapubic pain. She had urinary frequency, hesitancy, urgency, and urge incontinence. On physical examination, a right-sided abdominal mass was palpated. A computed tomography (CT) scan of the abdomen and pelvis was obtained. This revealed a large cystic fluid collection emanating from the right hip and extending into the right hemipelvis (Figures 1 \& 2). The mass was large enough to displace the right ureter and bladder, resulting in mild hydroureter, borderline hydronephrosis, and thickening of the bladder wall (Figure 3). A urinalysis showed 11-20 red blood cells per high-powered field. A urine culture was negative. The urinary cytology was negative for malignant cells. Further evaluation consisted of a cystoscopy and rightsided retrograde pyelogram. The cystoscopy revealed roughened bladder mucosa on the right side of the bladder wall and no bladder tumors. A bladder biopsy was negative for malignancy. The retrograde pyelogram was significant for a medially displaced, but normal caliber, right-sided ureter with no significant hydronephrosis.

In reviewing the patient's past medical history, it was noted that she had a right hip arthroplasty performed in 1970. She had three subsequent revisions, the most recent of which was thirteen years prior to her recent presentation. The cyst was thought to be the sequelae of long-standing particle disease, resulting in inflammatory changes within the joint and erosion of the medial aspect of the right acetabulum. The collection was felt to be benign, and no operative intervention was recommended by the patient's orthopedic surgeon.

Interestingly, her symptoms resolved within a few months after her urologic evaluation. As she was asymptomatic with no signs of systemic infection and her urinary complaints had resolved, a conservative approach of simple observation of the mass was chosen. On follow-up pelvic ultrasound six months later, the mass remained stable.

\section{Discussion}

Urinary tract complaints are not unusual after total hip arthroplasty, with the most common problem being urinary retention [1]. These urinary complaints are often acute complications related to perioperative and postoperative management. However, urinary symptoms may occur at any time after hip arthroplasty. While uncommon, synovial cysts arising from prosthetic hip replacements can cause urinary complaints years after the initial operation [2-4]. These cysts may be the result of increased friction and particle debris within the joint. The friction and debris result in the release of inflammatory 

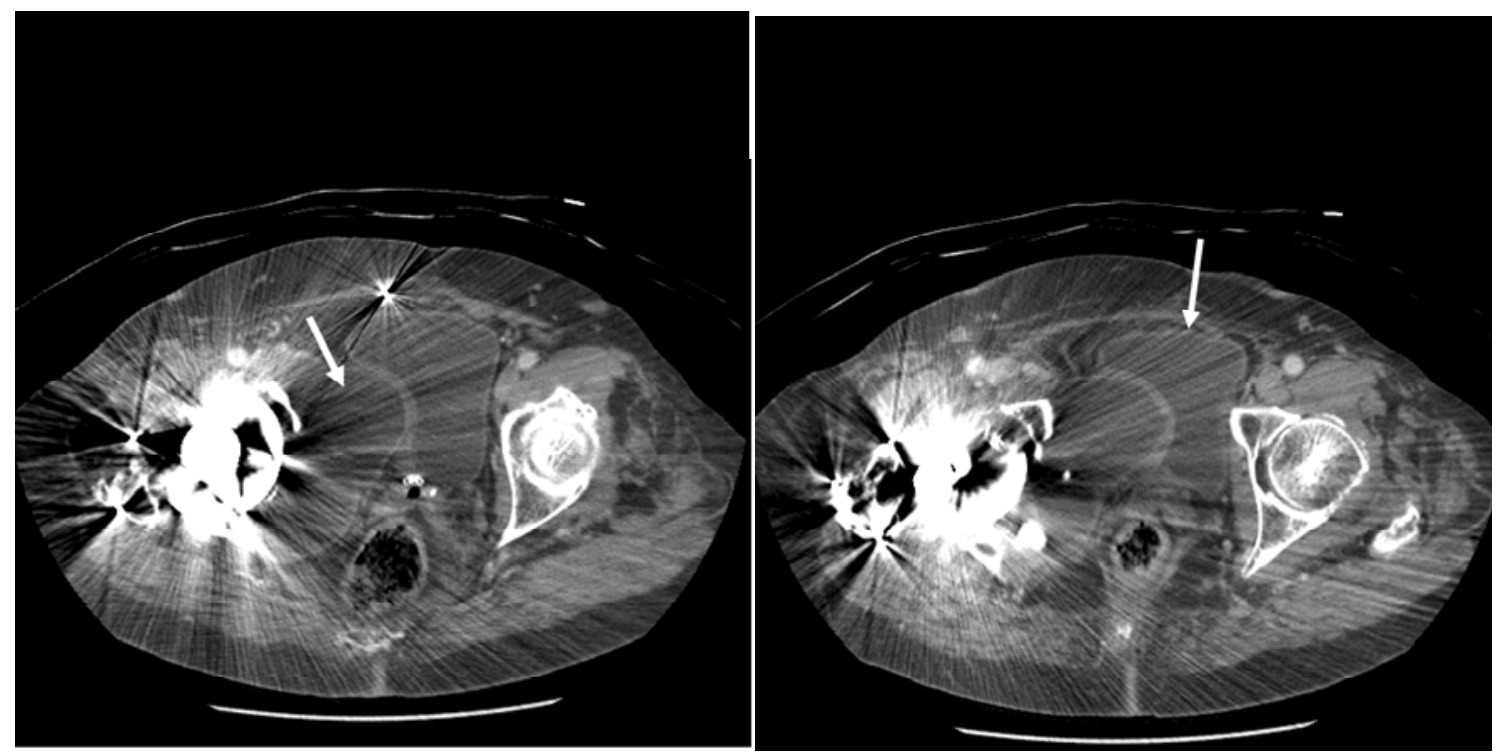

Figure 1 and Figure 2. CT scan images of the abdomen and pelvis revealing a large cystic fluid collection emanating from the right hip and extending into the right hemipelvis.

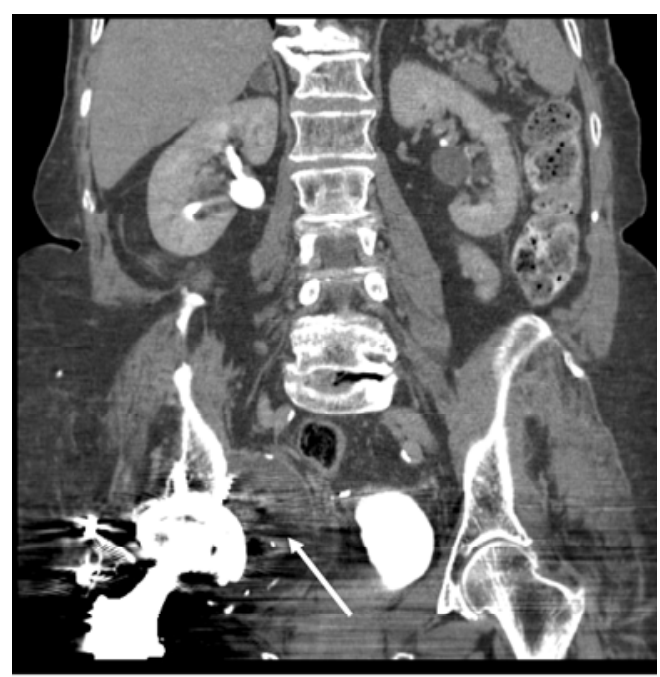

Figure 3. CT scan reveals a large mass displacing the right ureter and bladder, resulting in mild hydroureter, borderline hydronephrosis, and thickening of the bladder wall.

mediators which can cause osteolysis, resulting in an increased release of synovial fluid. Defects in the acetabulum allow fluid to escape from the joint space, thus forming a cyst [2-5]. Extrusion of cement into the pelvis may allow particles of polyethylene wear to migrate into the pelvis and cause cyst formation [4]. Cysts may form in the groin, pelvis, or retroperitoneum. These cysts can potentially cause compression of the colon, ureters, bladder, uterus, vagina, and neurovascular structures [2, 4-6]. Treatment options include observation, aspiration, excision, and revision of the total hip arthroplasty.
Although acute urinary tract complaints such as urinary retention after hip arthroplasty are common [1], those patients presenting with symptoms years after hip arthroplasty warrant a thorough evaluation. The physical exam was instrumental in this case, as it identified the abdominal mass which, through imaging resulted in the correct diagnosis. This case also demonstrates that in the absence of symptoms successful non-operative treatment of synovial cysts arising from a prosthetic hip is possible.

\section{Acknowledgement}

The authors thank the Marshfield Clinic Research Foundation's Office of Scientific Writing and Publication for editorial assistance with this manuscript.

\section{References}

[1] J. P. Izard, R. D. Sowery, M. T. Jaeger, and D. R. Siemens, "Parameters Affecting Urologic Complications after Major Joint Replacement Surgery," Canadian Journal of Urology, Vol. 13, No. 3, June 2006, pp. 3158-3163.

[2] R. D. DeFrang, W. D. Guyer, J. M. Porter, and P. J. Duwelius, "Synovial Cyst Formation Complicating Total Hip Arthroplasty: A Case Report," Clinical Orthopedics and Related Research, No. 325, April 1996, pp. 163-167.

[3] A. Nehme, D. A. Oakes, B. Marcheix, A. Gomez-Brouchet, and J. Puget, "Compressive Intrapelvic Synovial Cysts: An Early Complication of an HA-Coated Cup," Clinical Orthopedics and Re- 
lated Research, No. 430, January 2005, pp. 232-236.

[4] E. Thienpont and H. Vernaeve, "Reactive Pelvic Cyst Following Total Hip Arthroplasty. A Case Report.” Acta Orthopaedica Belgica, Vol. 69, No. 3, June 2003, pp. 292-294.

[5] S. Madan, R. L. Jowett, and M. I. Goodwin, "Recurrent Intrapelvic Cyst Complicating
Metal-on-Metal Cemented Total Hip Arthroplasty,” Archives of Orthopaedic and Trauma Surgery, Vol.120, No. 9, 2000, pp. 508-510.

[6] R. W. McGraw, K. S. Morton, and C. P. Duncan, "Massive Intrapelvic Synovial Cyst as a Complication of Total Hip Replacement Arthroplasty: A Case Report," Canadian Journal of Surgery, Vol. 34, No. 3, June 1991, pp. 267-269. 